\title{
Spatio-Temporal Database and Its Models: A Review
}

\author{
Rainu Nandal \\ University Institute of Engineering and Technology, M.D. University, India
}

\begin{abstract}
This paper focus on the study of spatial temporal database and its models and different types of applications where dynamic modeling of spatial temporal database can be used, this is an emerging field which places lot of contribution in DBMS with the aspects of the real world. Spatio-Temporal data models are the heart of a Spatio-Temporal Information System (STIS); they describe object data types, relationships, operations and rules to maintain database integrity. A rigorous data model must anticipate spatio-temporal queries and analytical methods to be performed in the STIS. Spatio-temporal database models are proposed to deal with real world applications, where spatial changes occur over the time line. A serious weakness of existing models is that each of them deals with few common characteristics found across a number of specific applications. Thus the applicability of the model to different cases, fails on spatio-temporal behaviors not anticipated by the application used for the initial model development.
\end{abstract}

Keywords-Land Information System (LIS), Geographical Information System (GIS), Spatial-temporal databases, STIS.

\section{Introduction}

Traditional databases are organized by fields, records, and files. A field is a single piece of information; a record is one complete set of fields; and a file is a collection of records. For example, a telephone book is equivalent to a file. It contains a list of records, each of which consists of three fields: name, address, and telephone number. A database is a collection of information that is organized so that it can easily be accessed, managed, and updated. In one view, databases can be classified according to types of content: bibliographic, full-text, numeric, and images. In computing, databases are sometimes classified according to their organizational approach. [4]

The type of database system that you require depends on a number of factors, such as:

- the complexity of the data involved, e.g. plain text, images, sound files.

- the quantity of data to be stored and processed.

- whether the data needs to be accessed and amended by more than one person simultaneously.

- whether data needs to be imported from, or exported to, other IT systems.[7]

The software programs for databases create one of four common types: hierarchical databases, network databases, relational databases or object-oriented databases and two more types: spatial databases and temporal databases.

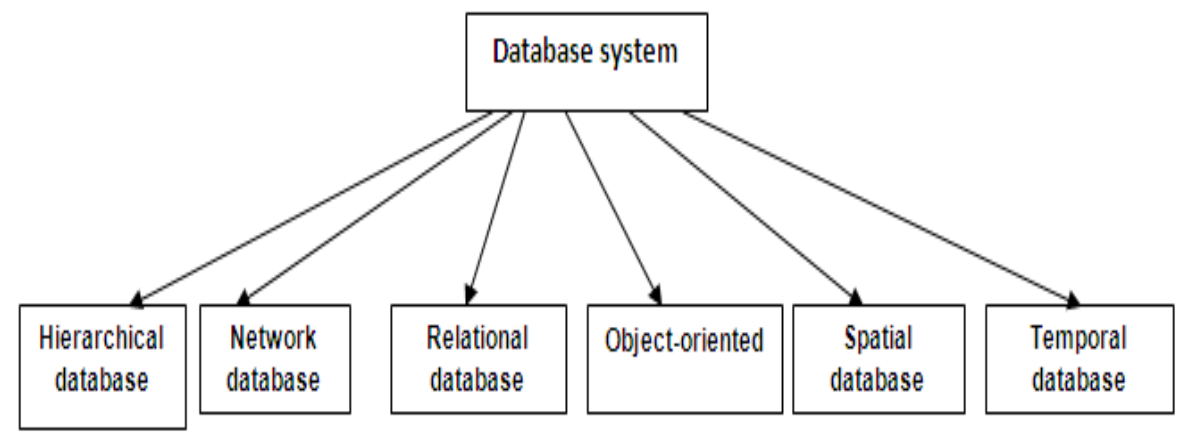

Fig 1: Types of databases

\subsection{Hierarchical database}

The hierarchical database is one of the oldest types of database management systems. It is most commonly used on mainframe computers. The database creator pre-defines the relationships between each record and its data. The structure requires a root record, or parent, from which the database designer creates a parent-child relationship for each bit of data that goes into the database. [7] 


\subsection{Network database}

A network database also organizes data by using defined parent-child relationships. Like a real family, the network database structure allows a piece of data classified as a child to have more than one parent. This is an improvement over hierarchical types of database management systems. It allows users to connect information in one database to another set of data through the parent record and the child record. [7]

\subsection{Relational database}

The relational database management system has increased in popularity because of its flexibility and ease of use. It allows the database designer to use individual pieces of information to create relationships between separate databases without the restriction of parent or owner relationships. The information in one database that ties it to data in a different one is a unique identifier, such as an employee identification number. [7]

\subsection{Object-oriented database}

Object-oriented types of database management systems provide a way to organize data other than numbers and text. Designers use them to accommodate multimedia items such as photos, music and videos. This database management system uses two identifiers for each item. The first is a descriptive object name, and the second is a miniature program with instructions or methods that the computer runs during storage and retrieval. The two parts become an object that the database users can organize like they can with text or numbers. [7]

\subsection{Spatial database}

A spatial database is a database that is optimized to store and query data that is related to objects in space, including points, lines and polygons. It offers spatial data types and stores information relating to geometric or geographical space. The spatial database stores a collection of space related data. [15]

\subsection{Temporal database}

Temporal database stores data relating to time instance. It offers temporal data types and stores information relating to past, present and future e.g. the history of the stock market or the movement of the employee with an organization. Thus a temporal database stores a collection of time related data. [4]From the above listed six database models, the two models namely: Temporal database, Spatial database will be used in the present work. The descriptions of these two models are as explained below.

\section{Spatial Database}

A spatial database is a database that is optimized to store and query data that is related to objects in space, including points, lines and polygons. While typical databases can understand various numeric and character types of data, additional functionality needs to be added for databases to process spatial data types. These are typically called geometry or feature. Spatial database management systems aim at the effective and efficient management of data related to [1][18].

- a space such as the physical world (geography, urban planning, astronomy);

- parts of living organisms (anatomy of the human body);

- engineering design (very large scale integrated circuits, the design of an automobile, or the molecular structure of a pharmaceutical drug); and

- conceptual information space (a multidimensional decision support system, fluid flow, or an electromagnetic field).[24]

Commercial examples of spatial database management include Informix's spatial data-blades (i.e., 2D, 3D, Geodetic), Oracle's Universal server with either Spatial Data Option or Spatial Data Cartridge and ESRI's Spatial Data Engine (SDE). Research prototype examples of spatial database management systems include spatial data blades with Postgres, Predator, and Paradise. The functionalities provided by these systems include a set of spatial datatypes such as a point, line-segment and polygon, and a set of spatial operations such as inside, intersection, and distance. The spatial types and operations may be made part of a query language such as SQL, which allows spatial querying when combined with an object-relational database management system. The performance enhancement provided by these systems includes a multidimensional spatial index and algorithms for spatial access methods, spatial range queries, and spatial joins. Spatial indexing with concurrency control may be implemented in the object-relational server for performance reasons. Existing and emerging applications require new functionalities including the modeling of network spaces and continuous fields. The performance needs of emerging applications require not only the management of large data sets, but also new processing strategies for spatial set operations, field operations (e.g., slope), and network analysis (e.g., shortest-path, routeevaluation).[18] 


\section{Temporal Database}

Commercial database management systems (DBMS) such as Oracle, Sybase, Informix and O2 allow the storage of huge amounts of data. This data is usually considered to be valid now. Past or future data is not stored. Past data refers to data which was stored in the database at an earlier time instant and which might has been modified or deleted in the meantime. Past data usually is overwritten with new (updated) data. Future data refers to data which is considered to be valid at a future time instant (but not now).[25]Temporal data stored in a temporal database is different from the data stored in non-temporal database in that a time period attached to the data expresses when it was valid or stored in the database. As mentioned above, conventional databases consider the data stored in it to be valid at time instant now, they do not keep track of past or future database states. By attaching a time period to the data, it becomes possible to store different database states. A first step towards a temporal database thus is to timestamp the data. This allows the distinction of different database states. One approach is that a temporal database may timestamp entities with time periods. Another approach is the timestamping of the property values of the entities. In the relational data model, tuples are timestamped, where as in object-oriented data models, objects and/or attribute values may be timestamped.[2] What time period do we store in these timestamps? There are mainly two different notions of time which are relevant for temporal databases. One is called the valid time, the other one is the transaction time. Valid time denotes the time period during which a fact is true with respect to the real world. Transaction time is the time period during which a fact is stored in the database. Note that these two time periods do not have to be the same for a single fact. [3][4] To categorize temporal data, one can adopt different criteria. Below we list several commonly used categorizations for temporal data:[16][8]

Partially Temporal vs. Fully Temporal: - A temporal dataset is partially temporal if it contains data items whose temporal relationships such as before and after are undecidable. For instance, web log is partially temporal, as it is often impossible to decide the exact access time to the same web page from different web sessions. In contrast, in a fully temporal dataset, the temporal relationship between every pair of data items is decidable:-

Regularly Timestamped vs. Irregularly Timestamped:- In regularly timestamped data, measurements are recorded at equal-spaced time points. Otherwise, the data is irregularly timestamped.

Univariate vs. Multivariate:- Univariate temporal data describes the temporal behavior of one variable. Multivariate temporal data on the other hand describes the temporal behavior of more than one variable.

Uni-subject vs. Multi-subject:- A uni-subject temporal data involves only one subject. Whereas a multisubject temporal data involves more than one subject

\section{Spatio-Temporal Database}

Spatio-temporal databases deal with applications where data types are characterized by both spatial and temporal semantics. Spatio-temporal data handling was not a straightforward task due to the complexity of the data structures requiring careful analysis in structuring the dimensions, together with the representation and manipulation of the data involved. Research on space-time representation has focused on a number of specific areas, including: (a) the ontology of space and time and the development of efficient and robust space-time database models and languages; (b) inexactness and scaling issues; (c) graphical user interfaces and query optimization; (d) indexing techniques for space-time databases.[5][24]

\section{Spatio-Temporal Data Modeling}

The ideas behind the spatio-temporal modeling can be broadly cross-classified according to: (a) their motivation, (b) their underlying objectives and (c) the scale of data. Under (a) the motivations for models can be classified into four classes: (i) extensions of time series methods to space (ii) extension of random field and imaging techniques to time (iii) interaction of time and space methods and (iv) physical models. Under (b) the main objectives can be viewed as either data reduction or prediction. Finally, under (c) the available data might be sparse or dense in time or space respectively, and the modeling approach often takes this scale of data into account.[6] Spatio-Temporal data models are the core of a Spatio-Temporal Information System (STIS); they define object data types, relationships, operations and rules to maintain database integrity. A rigorous data model must anticipate spatio-temporal queries and analytical methods to be performed in the STIS. Spatiotemporal database models are intended to deal with real world applications, where spatial changes occur over the time line. A serious weakness of existing models is that each of them deals with few common characteristics found across a number of specific applications. Thus the applicability of the model to different cases, fails on spatio-temporal behaviors not anticipated by the application used for the initial model development. [24]Several different forms of spatio-temporal data types are available in real applications. While they all share the availability of some kind of spatial and temporal aspects, the extent of such information and the way they are 
related can combine to several different kinds of data objects. Figure 1 visually depicts a possible classification of such data types, based on two dimensions:[20][23]

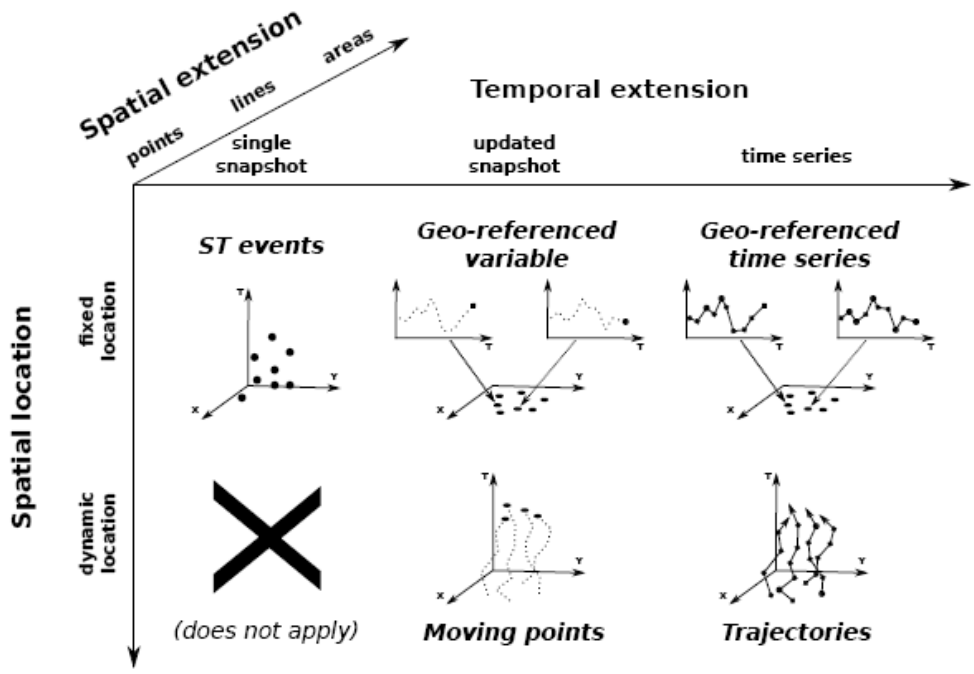

Fig 5.1: Spatio-temporal data types dimensions[19]

- the temporal dimension describes to which extent the evolution of the object is captured by the data. The very basic case consists of objects that do not evolve at all, in which case only a static snapshot view of each object is available. In slightly more complex contexts, each object can change its status, yet only its most recent value (i.e., an updated snapshot) is known, therefore without any knowledge about its past history. Finally, we can have the extreme case where the full history of the object is kept, thus forming a time series of the status it reversed;

- the spatial dimension describes whether the objects considered are associated to a fixed location (e.g., the information collected by sensors fixed to the ground) or they can move, i.e., their location is dynamic and can change in time. In addition to these two dimensions, a third, auxiliary one is mentioned in our classification, which is related to the spatial extension of the objects involved. The simplest case, which is also the most popular in real world case studies, considers point-wise objects, while more complex ones can take into consideration objects with a extension, such as lines and areas.

\section{Spatio-Temporal Data Models}

Throughout the relatively young history of research on spatio-temporal modeling, a substantial numbers of models have been presented. Spatio-temporal data models are classified into the following ten categories:[12][14][20][10]

\subsection{The Snapshot Model}

One of the simplest spatio-temporal data models is the snapshot model. Temporal information has been incorporated into this spatial data model by time-stamping layers. In this model, every layer is a collection of temporally homogeneous units of one theme. It shows the states of a geographic distribution at different times without explicit temporal relations among layers.

\subsection{The Space-Time Composite (STC) Data Model}

The method is brought forward by Chrisman to the vector model in 1983. It is based on the principle that every line in space and time is projected down to the spatial plane and intersected with each other creating a polygon mesh. Each polygon in this mesh has its own attribute history associated with it. Each new amendment is intersected with the already existing lines, and new polygons are formed with individual histories. Its biggest weakness lies in polygon broken and depend on related database excessively.

\subsection{Data Models based on Simple Time-Stamping}

In this model, to tag every object with a pair of timestamps, one for the time of creation and one for the time of cessation. Current objects have their cessation time given by a special value "NOW", "CURRENT", or "NULL" The model is based on the linear, discrete, absolute time model. Only valid time is supported while the 
model supports multiple granularities. Time is represented as an attribute of the object and vector structure of space is assumed.

\subsection{Event-Oriented Models}

In the space time model based on affairs, the state change of the space time object is sprung by geography things. Passing through import affairs table, putting attributes or space change record in the each module of the same affair, showing, giving the describe method of time in sequence, which can build up the relation of object state and geography thing, In order to provide the foundation of the tense operation for high level. The space time model based on the affairs is very fit to the query of this question as "What happened in some times and in some areas", and also have good consistency in data and less fraction redundancy degree of the data.

\subsection{The Three-Domain Model}

This model represents semantics, space and time separately and provides links between them to describe geographic processes and phenomena. The semantic domain holds uniquely identifiable objects that correspond to human concepts independent of their spatial and temporal location. It identifies semantics, spatial domain, and, temporal domain for spatio-temporal data. The links between space and time are described through different semantics

\subsection{The History Graph Model}

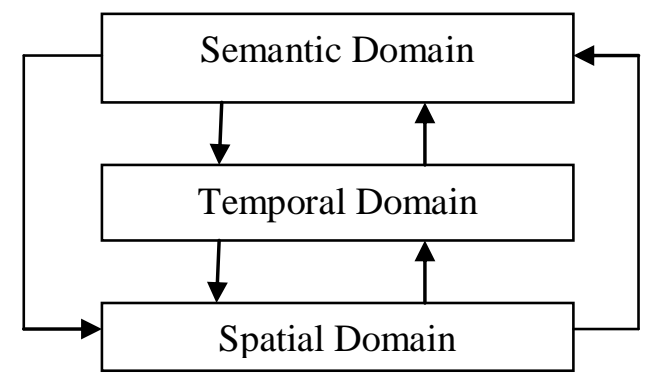

Fig-6.1: The three-domain model

The history graph model is to identify all types of temporal behavior and to manage both objects and events. The intention of the history graph notation is to visualize the temporal element of geographical and other information. It is based on the simple idea that an object may either be in a static, a changing or a ceased state. In the history graph notation, the static states called object versions are shown with rectangular boxes, while the changing states called transitions between versions are shown with round ended boxes (or circles in case of sudden changes).

\subsection{The Spatio-Temporal Entity-Relationship (STER) Model}

The careful analysis of spatio-temporal applications and behavior of spatial and temporal entities suggested that entity sets with their attributes and relationships could capture the dynamic nature of spatiotemporal databases. In STER model, three types of time aspects can be defined: (i) valid time, (ii) transaction time, and (iii) existence time. The valid time of a fact is the time when the fact is true in the modeled reality. The transaction time of a geo database is the time when the element is the part of the current state of the geo database. The transaction time is applied not only to facts but to any element that may be stored in a database. The existence time refers to the time when the object exists.

\subsection{Object-Relationship (O-R) Model}

The implementation of object-relationship models describe "processes, which act on the geometric attributes of an entity" and illustrate the importance of capturing the processes, which cause change in connection with space and time.

\subsection{Spatio-Temporal Object-Oriented (O-O) Data Models}

This model organizes geography space time with the object oriented idea. Among them object is the independent pack which is a concept entity have only one marking. Each geography space time object encapsulate the tense characteristic, space characteristic, attribute characteristic ,related behavior operation and the relation with other objects.

The model introduces the concept of version management in order to integrate object and event elements. Two main versioning levels can be distinguished: object version and object configuration. Four basic premises underlie the proposed model at the object version level: a) Every object must have an initial version, b) 
a hierarchical structure is imposed on the versions of an object, c) different versions of an object denote different object instances, d) among versions, a current version is always distinguished.

\subsection{Moving Object Data Models}

The moving object data model, where spatio-temporal data is abstracted as a collection of moving objects including points and regions. It models time as an integral part of spatial entities and captures both changes and movements.

\section{Features Of Spatio-Temporal Data Model}

The above spatio-temporal database models vary on completeness, formalization and implementation. These data models are describe with the following features in table 7.1[14] :-

- Formalisation: This factor shows if the model has been formally defined or not.

- Implementation: This criterion depicts if the model has been implemented or not.

- Tool: If the model has been implemented, the name of the tool developed is listed here.

- Application: The case study used to analyse and develop the model.

- Spatial model: The spatial model used as the basis for the development of the corresponding spatiotemporal model.

- Temporal model: The temporal model used to define and develop the spatio-temporal model.

\begin{tabular}{|c|c|c|c|c|c|c|}
\hline $\begin{array}{l}\text { Spatio- } \\
\text { Temporal } \\
\text { datamodels }\end{array}$ & $\begin{array}{l}\text { Formalisatio } \\
\mathrm{n}\end{array}$ & $\begin{array}{l}\text { Implementatio } \\
\mathrm{n}\end{array}$ & Tool & Application & Spatial Model & $\begin{array}{l}\text { Temporal } \\
\text { Model }\end{array}$ \\
\hline Snapshot & No & No & None & LIS & GIS & N/A \\
\hline STC & No & No & None & LIS & GIS & N/A \\
\hline $\begin{array}{l}\text { Simple Time } \\
\text { Stamping }\end{array}$ & No & No & None & $\begin{array}{l}\text { Historical } \\
\text { cadastral } \\
\text { database }\end{array}$ & $\begin{array}{l}\text { US spatial } \\
\text { data transfer } \\
\text { standard }\end{array}$ & N/A \\
\hline $\begin{array}{l}\text { Event } \\
\text { Oriented }\end{array}$ & Yes & Yes & TEMPEST & LIS & $\begin{array}{l}\text { GIS with } \\
\text { event dates }\end{array}$ & $\begin{array}{l}\text { Ordered } \\
\text { time } \\
\text { models }\end{array}$ \\
\hline 3-Domain & Yes & No & None & LIS & $\begin{array}{l}\text { Relational } \\
\text { spatial } \\
\text { database }\end{array}$ & $\begin{array}{l}\text { Relational } \\
\text { version } \\
\text { tables }\end{array}$ \\
\hline History Graph & No & No & None & LIS & N/A & Graphs \\
\hline STER & Yes & No & None & $\begin{array}{l}\text { Cadastral } \\
\text { application }\end{array}$ & $\begin{array}{l}\text { Spatial } \\
\text { indicator }\end{array}$ & $\begin{array}{l}\text { Temporal } \\
\text { indicators }\end{array}$ \\
\hline O-R & Yes & Yes & MADS & $\begin{array}{l}\text { Rural urban } \\
\text { land use } \\
\text { application }\end{array}$ & MEOSIG & $\begin{array}{l}\text { MODUL- } \\
\mathrm{R} \\
\text { POLLEN } \\
\end{array}$ \\
\hline $\mathrm{O}-\mathrm{O}$ & Yes & Yes & Geo-OM & LIS & ERT model & $\begin{array}{l}\text { Temporal } \\
\text { base } \\
\text { model }\end{array}$ \\
\hline STUML & Yes & No & None & $\begin{array}{l}\text { Regional } \\
\text { health care } \\
\text { example }\end{array}$ & $\begin{array}{l}\text { Spatial } \\
\text { indicator }\end{array}$ & $\begin{array}{l}\text { Temporal } \\
\text { indicators }\end{array}$ \\
\hline $\begin{array}{l}\text { Moving } \\
\text { Objects }\end{array}$ & Yes & Yes & $\begin{array}{l}\text { SECONDO } \\
\text { module }\end{array}$ & $\begin{array}{l}\text { Multimedia } \\
\text { scenario, } \\
\text { Forestfire } \\
\text { control } \\
\text { management }\end{array}$ & $\begin{array}{l}\text { Abstraction of } \\
\text { spatial data } \\
\text { types \& } \\
\text { Oracle Spatial }\end{array}$ & $\begin{array}{l}\text { Abstractio } \\
\mathrm{n} \text { of } \\
\text { temporal } \\
\text { data types } \\
\text { \&TAU } \\
\text { types }\end{array}$ \\
\hline
\end{tabular}

Table7.1: Comparing existing spatio-temporal data models

VIII. Practical project applications for spatio-temporal modeling of dynamic phenomena in gis

A temporal GIS has three general components (temporal database, temporal visualization and temporal analysis) that lead to three research domain in temporal GIS.Animation as an efficient approach for representation of temporal changes is implemented and then optimum path analysis are extended as it can handle time dependent graphical and attribute information on the base of the animation representation method. 
Integration of temporal path analysis with animation can be useful in many cases such as more reliable decision making and faster and better access to emergency services in critical conditions is possible if traffic volume and road network conditions imported into GIS databases, dynamically.[28]With this integration of animation and optimum path analysis, the system can obtain real optimum path with consideration of momentary traffic volume of road networks and other temporal parameters that affect selection of optimum path. If this system to be integrated with a GIS-GPS system, it can handle real time position of vehicle in order to evaluate real optimum path for each moving vehicle.[17][11]The major applications of Spatio-temporal modeling of dynamic phenomena in GIS are as given below:[21][22][9]

\subsection{Roads traffic volume simulation}

In practice, roads traffic volumes have to be collected and transmitted to GIS database with specific sensors. These sensors monitor and record traffic volume changes and then apply these changes to the database. Using such data, the simulations can be achieved for analysis of the traffic volumes.

\subsection{Vehicle movement simulation}

Vehicle movements in a GIS can be considered as changes of planimetric position attribute in the database. This position's attributes (X, Y / Phi, Lambda coordinates) can be obtained from GPS or other positioning systems and then transmitted to the database. Vehicle movements with respect to road networks can be simulated henceforth, and then resulted attributes are applied to the database.

\subsection{Graphical changes modeling}

In order to represent and model graphical changes related to road networks in a cadastral map at a scale, for example, 1:500, the snapshot model can be used. For this purpose, every change is stored in a separate layer (next changes can be added to model with storage of new snapshots in the specified path on computer), then the implemented system represents these snapshots sequentially with the animation method.

\subsection{Extended optimum path analysis}

In common cases this analysis requires only one start point, one end point, some intermediate points and one attribute field such as traffic volume that includes cost information for each segment. However, in a temporal GIS, each of these elements can change over time (movement of start, end and intermediate points and change in attribute field or any combination of them). In addition to, graphical information can have structural changes (such as construction of new roads). Therefore, optimum path analysis must be extended in order to handle these temporal changes. Moreover, representation of temporal optimum path must be coordinated with animation picture rate.

\subsection{Animation}

In graphical images such as GIF files that nowadays are frequently used to implement animation under web applications, there are some images that aligned sequentially in a file and are shown one by one. In this approach there are no tools for users to control picture rate or analysis on images. But the animation that is subjected in GIS, must present the ability to use all GIS analysis, querying, and all other static GIS capabilities. When animation is used in GIS, there is an important term of picture rate that returns to representation period versus real period of occurrence events. For example representation of annual period of sea level changes in a few minutes or an accident moment in larger duration of time may be considered. Thus each GIS, which use animation, must prepare the ability to control picture rate, too.

In the system implemented in this research, there are some abilities to represent graphical changes in addition to representation of database changes with animation. In this case, user must first store new snapshots in a path on hard disk and then this path, number of snapshots and number of loops that are required; have to be introduced to the system. Figure 4 represents a schema of the implemented system.[29] 


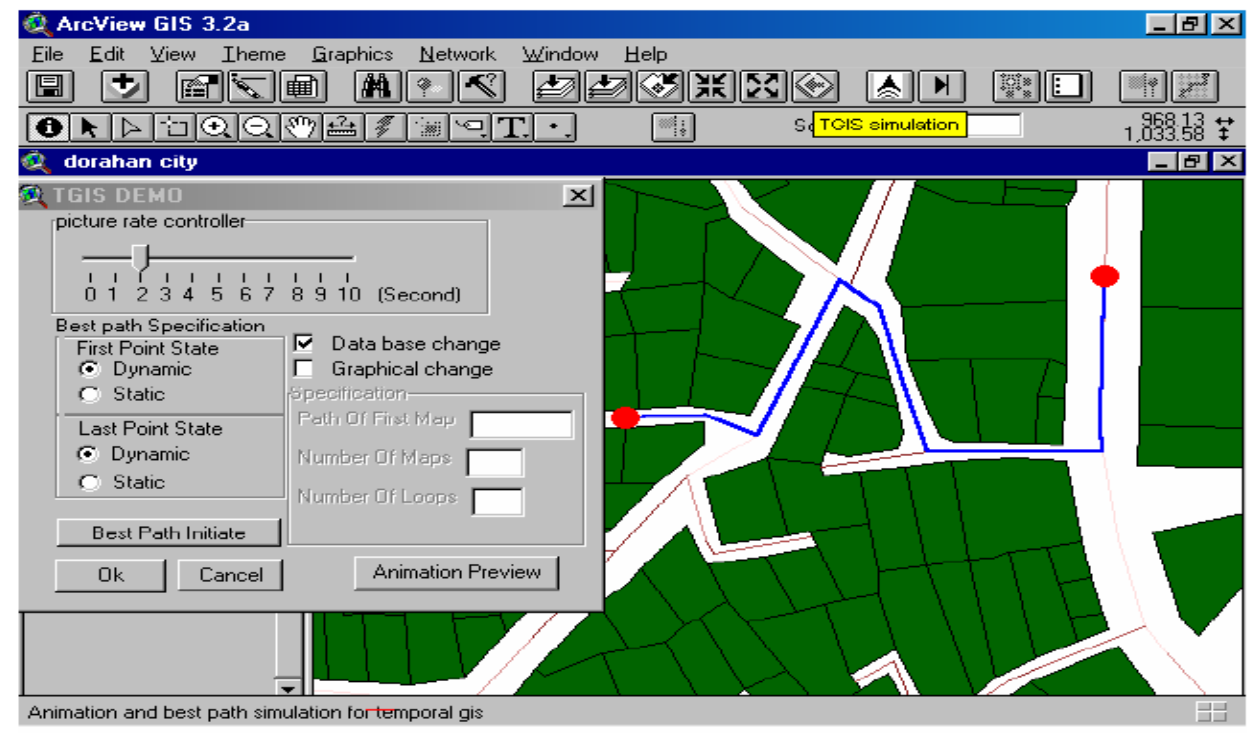

Fig. 8.1- A schema of implemented system interface[13]

\section{Research Issues In Spatio-Temporal Database}

The following points consider in depth the spatio-temporal research agenda that is important in the framework of computing science, in terms of the necessities that find out the priorities of modeling spatio-temporal applications and highlight key issues from those of lesser significance:

- Spatio-temporal databases typically deal with large complex bodies of spatially referenced data, which is required to be readily available. As such indexing techniques for space-time databases and more specifically for real-time applications that describe continuously evolving spatial entities; are still an important and open research area.[30]

- A sizeable proportion of the data is either regularly/irregularly updated from external data sources or need to be continuously updated due to evolution of natural processes.[35]

- Some of the data are noisy, conflicting and incomplete. More analytically, a major problem with spatial data is the control of error propagation under spatial operations. Further research is needed on finite precision geometry and multiple resolution techniques.[31]

- Complex functions and calculations involving operators, relationship status and objects for the prediction of their future motion need to be designed. Research is also needed to be carried out on applications of newer computational paradigms, such as constraint-based approaches, fuzzy sets and rough sets.[33]

- A growing number of researchers in both the DBMS and GIS communities have come to the realization that a general, application-independent solution that allows an optimal combination of simplicity, flexibility and efficiency will require rethinking at an abstract level and new types of implementation data models and associated query languages. This solution needs to be based upon a uniform ontological framework and requires a multi-representational approach.[32][34]

\section{Conclusion}

The spatial and temporal dimensions should be considered separately and incorporated into the database design. Modeling of Temporal and spatial characteristics captures the aspects of the real world. The thematic characteristics of spatio-temporal objects should be identified and modeled together with the spatial and temporal characteristics. The study is done in the real world application which brings out new directions and requirements for further developments. The spatio-temporal models are more concerned with theoretical notation of spatio- temporal data. In future, the next step in spatio-temporal database development is the testing stage where the models proposed are implemented on different applications to identify further requirements and research directions. 


\section{REFERENCES}

[1]. A. Renolen, "Temporal Maps and Temporal Geographical Information Systems (Review of Research)”, Department of Surveying and Mapping, The Norwegian Institute of Technology, 1997.

[2]. A.G. Cohn and S.M. Hazarika. "Qualitative spatial representation and reasoning: An overview", Fundamental Informatics, 46(12): $1-29,2001$

[3]. Auroop R Ganguly \& Karsten Steinhaeuser, (2008) "Data Mining for Climate Change and Impacts", IEEE International conference on Data mining workshop, ICDMW, 385-394, 15-19 Dec, Italy.

[4]. Bitner T, (2000) "Rough sets in Spatiotemporal data mining", Proceedings of International workshop on Temporal, Spatial and Spatiotemporal Data Mining, Lyon, France.

[5]. Bogorny V \& Shekhar S, (2010) "Spatial and Spatio-Temporal Data Mining", IEEE $10^{\text {th }}$ International Conference on Data Mining (ICDM), Sydney,NSW.

[6]. Bruno De C. Leal et.al, (2011) “From Conceptual Modeling to Logical Representation of Trajectories in SGBDOR and DW Systems", Journal of Information and Data Management, Vol 2, No 3.

[7]. C. Armenakis, "Estimation and Organization of Spatio-Temporal Data", Proceedings of the Canadian Conference on GIS92, Ottawa, Canada, 1992.

[8]. C. Bettini, X. S. Wang, and S. Jajodia, "A general framework and reasoning models for time granularity", Proceedings of Third International Workshop on Temporal Representation and reasoning (TIME '96), pp. 104-11,1996.

[9]. C.S. Jensen, J. Clifford, R. Elmasri, S.K. Gadia, P. Hayes and S. Jajodia, eds., "A Glossary of Temporal Database Concepts" ACM SIGMOD Record, vol. 23, no. 1, pp. 52-64, Mar. 1994.

[10]. Changbin Wu, (2011) "Detecting Spatio-Temporal Topological relationships between boundary lines of parcel", International Conference on Remote sensing, Environment and Transportation Engineering, Nanjing.

[11]. D. Peuquet, "Making Space for Time: Issues in Spase-Time Data Representation" GeoInformatica, 5: 11-32, 2001.

[12]. Derya Birant \& Alp Kut, (2007) "ST-DBSCAN: An algorithm for clustering spatio-temporal data“, Data \& Knowledge Engineering, Volume 60, Issue 1, January, Pages 208-221.

[13]. F. Wang, G.B. Hall, and Subaryono, "Fuzzy Information Representation and Processing in Conventional GIS Software: Database Design and Application", International Journal of Geographical Information Systems, Vol. 4: 261-283, 1990.

[14]. Florian Verhein \& Sanjay Chawla, (2005) "Mining Spatio-Temporal Association Ruls, Sources, Sinks, Stationary Regions and Thoroughfares in Object Mobility Databases“, Technical Report Number 574, The University of Sydney.

[15]. Gabriel Pestana etal, (2005) "Multidimensional Modeling based on spatial,Temporal and Spatio-Temporal Stereotypes“, ESRI International User Conference, July, Sandiego, Califonia.

[16]. Gabriel Pestana, Miguel Mira da Silva,"Multidimensional Modeling based on Spatial, Temporal and Spatio-Temporal Stereotypes",ESRI International User Conference July 25-29, 2005

[17]. H. Yang, K. Marsolo, S. Parthasarathy, and S. Mehta. "Discovering spatial relationships between approximately equivalent patterns". In BIOKDD04: Workshop on Data Mining in Bioinfomatics (with SIGKDD04 Conf.), August 2004

[18]. H.F. Korth and A. Silberschatz, Database System Concepts.McGraw-Hill Advanced Computer Science Series. McGraw-HillBook Co., 1986

[19]. J M Kang, S Shekar, M Henjum, P Novak \& W Arnold, (2009) “ Discovering Teleconnected Flow Anomalies : A Relationship Analysis of Spatiotemporal Dynamic Neighborhoods”, In Symposium of Spatial and Temporal Databases SSTD’09, July 8-10, Aalborg, Denmark

[20]. James F Allen, "Time and Time Again: The Many Ways to Represent Time" Journal of Intelligent Systems, vol. 6, pp. 341-355, July 1991

[21]. Jia-Dong Ren, Jie Bao \& Hui-Yu Huang, (2003) "Research on Spatio-Temporal Data Model and Related Mining“, Proceeding of the Second International Conference on Machine Learning and Cybernetics, Xi'an, 2-5 November.

[22]. Jiawei Han, (2003) "Mining Spatiotemporal Knowledge: Methodologies and Research Issues", A position paper, KDV workshop

[23]. K.Venkateswara Rao, Dr. A.Govardhan \& Dr.K.V.Chalapati Rao, (2011) "Discovering Spatiotemporal Topological Relationships", The Second international workshop on Database Management Systems, DMS-2011, July, Chennai, India, Springer Proceedings LNCS-CCIS 198.

[24]. K.Venkateswara Rao, Dr. A.Govardhan \& Dr.K.V.Chalapati Rao, "Mining Topological Relationship Patterns from spatiotemporal Databases“, International Journal of Data Mining and Knowledge Management Process, IJDKP, Accepted.

[25]. M Ekram Azim et.al, (2011) “Detection of the Spatiotemporal Trends of Mercury in Lake Erie Fish Communities: A Bayesian Approach", ACS Environmental Science \& Technology, 45(6).

[26]. M. Erwig and M. Schneider, "Developments in Spatio-Temporal Query Languages", In IEEE Int.Workshop on Spatio-Temporal Data Models and Languages (STDML), pages 441-449, Florence,Italy, 1999.

[27]. M. Erwig and M. Schneider, "Spatio-Temporal Predicates", Technical Report 261, Fern Universitat Hagen, 1999. To appear in IEEE Transactions on Knowledge and Data Engineering

[28]. M. J. Egenhofer and J. R. Herring. "Spatial and Temporal Reasoning in Geographic Information Systems",Oxford University Press ,New York, 1998

[29]. M. Koubarakis, T. Sellis et al. (eds.), Spatio-Temporal Databases: The Chorochronos Approach, 2003. Springer-Verlag LNCS 2520.

[30]. M. Koubarakis, T.K. Sellis et al.(eds.),"Spatio-Temporal Databases: The Chorochronos Approach” Springer-Verlag LNCS 2520, 2003.

[31]. M. Yuan, "Temporal GIS and Spatio-Temporal Modeling " in Proceedings of Third International Conference on Integrating GIS and Environmental Modeling, Santa Fe, New Mexico, USA, 1996.

[32]. Manso J A, Times V C, Oliveira G, Alvares L \& Bogorny V, (2010) "DB-SMoT: A Direction based Spatio-Temporal Clustering Method", Fifth IEEE International Conference on intelligent Systems IEEE IS 2010.

[33]. Martin Erwig and Markus Schneider," Spatio-Temporal Predicates" IEEE TRANSACTIONS ON KNOWLEDGE AND DATA ENGINEERING, VOL. 14, NO. 4, JULY/AUGUST 2002881

[34]. Max J Egenhofer ," what is special about spatial database requirements for vehicles navigation in geographic space ", SIGMOD Rec.,vol.22,no. 2, pp.398-402,june 1993.

[35]. Michael Mcguire, Vandana J \& Aryya Gangopadhyay (2010) “ Spatiotemporal Neighborhood discovery for sensor data", In knowledge discovery from sensor data, Vol 5840.

[36]. N. Pelekis, B. Theodoulidis, I. Kopanakis, and Y. Theodoridis. "Literature review of spatio-temporal database models". Technical report, Center of Research in Information Management (CRIM), Department of Computation, UMIST; Department of Informatics, University of Piraeus, 2005. 
[37]. N. W. J. Hazelton, "Developments in Spatio-Temporal GIS", Proceedings of the First Regional Conference on GIS Research in Victoria and Tasmania, 1992.

[38]. O. Ahlqvist, J. Keukelaar and K. Oukbir, "Rough Classification and Accuracy Assessment", International Journal of Geographical Information Science, Vol. 14: 475-496, 2000.

[39]. O. Guenther and A. Buchmann. “Research Issues in Spatial Databases", SIGMOD Record, Vol. 19:61-68,1990.

[40]. O. Wolfson, B. Xu, S. Chamberlain, L. Jiang, "Moving Objects Databases: Issues and Solutions", Proceedings of the 10th Int. Conference on Scientific and Statistical Database Management, pages111-122, Capri, Italy, 1998.

[41]. P. J. Brockwell and R. A. Davis. "Introduction to Time Series and Forecasting”. Springer, ISBN: 0-387- 95351-5, 2003.

[42]. R.H. Guting, "An Introduction to Spatial Database Systems", VLDB Journal 4 (1994), 357-399.

[43]. Roberto Trasarti, Fabio Pinelli \& Mirco Nanni, (2011) “Mining Mobility User Profiles for Car Pooling“, KDD' 11, August 21-24, San Diego, California, USA.

[44]. S. Grumbach, P. Rigaux and L. Segoufin, "Spatio-Temporal Data Handling with Constraints", GeoInformatica, 5: 95-115, 2001.

[45]. S. Nadi and R.D. Mahmoud, "Spatio-Temporal Modeling of Dynamic Phenomena in GIS," in ScanGIS'2003 - The 9th Scandinavian Research Conference on GIS, 4-6 June 2003- Proceedings, Espoo, Finland , pp. 215- 225,2003.

[46]. S. Shekhar, S. Chawla, S. Ravada,A. Fetterer, X. Liu, C.T. Lu,"Spatial Databases: Accomplishments and Research Needs"

[47]. S.Kisilevich,F.Mansmann, M.Nanni, and S.Rinzivillo, "Spatio-temporal clustering," pp. 855-874, 2010

[48]. SUJIT K. SAHU, KANTI V. MARDIA,"Recent Trends in Modeling Spatio-temporal Data"

[49]. T. Abraham and J. F. Roddick. "Survey of spatio-temporal databases". Geoinformatica, 3(1):61-99, 1999.

[50]. Taher Omran \& Maryvonne, (2005) "Multidimensional Structures Dedicated to Continuous Spatiotemporal Phenomena", BNCOD 2005, LNCS 3567, pp 29-40.

[51]. Vieira M R, Frias Martinez V, Oliver N \& Frias Martinez E, (2010) "Characterizing Dense Urban Areas from Mobile Phone-call Data: Discovery and Social Dynamics", IEEE Second International Conference on Social Computing, Minneapolis, MN.

[52]. Viveca Asproth, Anita H.kansson, and Peter RÃ"vay, "Dynamic information in GIS systems," vol. 19, pp. 107 - 115, 1995

[53]. Xiaoyu Wang, Xiaofang Zhou and Sanglu Lu,"Spatio-temporal Data Modeling and management: A Survey"

[54]. Yan Huang, Cai Chen \& Pinliang Dong, (2008) "Modeling Herds and Their Evolvements from Trajectory Data", International Conference on Geographic Information Science, GISCIENCE. International Journal of Computer Science \& Engineering Survey (IJCSES) Vol.3, No.1, February 2012 